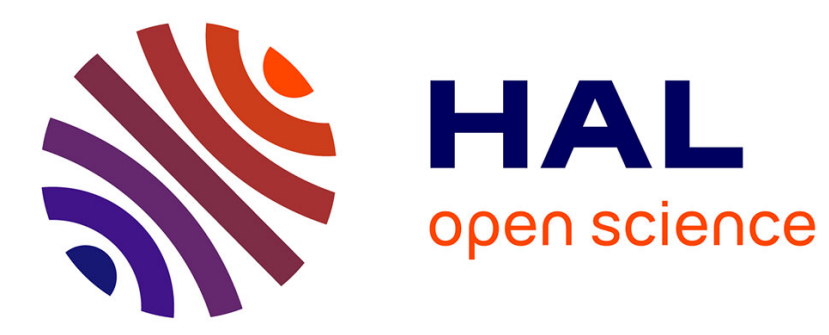

\title{
Calendrier et pouvoir à Rome: une double révolution
}

Béatrice Bakhouche

\section{To cite this version:}

Béatrice Bakhouche. Calendrier et pouvoir à Rome: une double révolution. Giornale Italiano di Filologia, 2016, 68, pp.89-119. 10.1484/J.GIF.5.112481 . hal-03174784

\section{HAL Id: hal-03174784 \\ https://hal.science/hal-03174784}

Submitted on 19 Mar 2021

HAL is a multi-disciplinary open access archive for the deposit and dissemination of scientific research documents, whether they are published or not. The documents may come from teaching and research institutions in France or abroad, or from public or private research centers.
L'archive ouverte pluridisciplinaire $\mathbf{H A L}$, est destinée au dépôt et à la diffusion de documents scientifiques de niveau recherche, publiés ou non, émanant des établissements d'enseignement et de recherche français ou étrangers, des laboratoires publics ou privés.

\section{(이)(\$)}

Distributed under a Creative Commons Attribution - NonCommercial - NoDerivatives| 4.0 


\section{Calendrier et pouvoir à Rome : une double révolution}

Résumé : On parle volontiers de 'réforme du calendrier' à propos de la modification du comput du temps par Jules César ; mais il serait plus judicieux de parler de révolution, tant les modifications rendent l'usage antérieur obsolète et instaurent une nouvelle ère, en même temps qu'ils élargissent au temps le pouvoir que le dictateur exerçait sur l'espace.

La division du mois, de son côté, subit elle aussi des transformations radicales dont la chronologie est généralement mal maîtrisée. Il s'agira ici de traquer les premières apparitions de la semaine et son intégration dans le calendrier.

Abstract : 'Calendar's reform' is usually said about the change of time** by Caesar ; but it would be better

Les Romains se sont beaucoup intéressés aux questions du temps, et par suite à celle du calendrier. Les 'antiquaires', les spécialistes d'agronomie ou d'astronomie, voire les encyclopédistes à partir de Varron, ont attaché une grande importance au comput en tant que système de division du temps prenant en compte les mouvements du Soleil et de la Lune mais aussi les jours fastes et ceux qui ne l'étaient pas, tandis que le temps constituait, dans son essence, un élément de la dialectique philosophique qui l'opposait à l'éternité ou à la pérennité, sempiternitas. Si les questions de calendrier ont été abordées par divers auteurs latins, en lien souvent avec les travaux des champs, seuls deux d'entre eux à date tardive Censorinus (III ${ }^{\mathrm{e}}$ s.) et Macrobe $\left(\mathrm{V}^{\mathrm{e}} \mathrm{s}\right.$.) - ont consacré des pages à l'histoire du calendrier. Avant eux, un historien romain de langue grec, Dion Cassius, avait consacré plusieurs chapitres de son Histoire romaine aux problèmes de calendrier, en marge de son sujet principal.

À côté de cette dimension intellectuelle, le calendrier est incontestablement un marqueur politique, culturel et cultuel. Loin d'être un simple outil, un instrument purement technique,

\footnotetext{
- Cet article est la version remaniée d'une communication que j'ai faite à Londres le 25 juin 2015 à l'University College London, au cours de la journée d'étude organisée par le Prof. Sacha Stern sur 'The origins of the seven-day week'.
} 
c'est un élément de la société, c'est au contraire un de ses éléments constitutifs ${ }^{1}$. C'est ainsi que le premier siècle de la république romaine connut une véritable révolution par la publication d'éléments juridiques et calendaires jusque là dans les seules mains des patriciens : Cnaeus Flavius en effet, secrétaire d'Appius Claudius Caecus, publia en 304 av. J.-C., à l'insu de son patronus, les formules religieuses permettant et encadrant les procédures juridiques et le calendrier, et donnant en même temps les jours où il est possible d'intenter un procès $^{2}$. Plus tard, en 189, M. Fulvius Nobilior a placé, dans le temps d'Hercule Musagète qu'il a fait bâtir à Rome, une copie des Fasti, peut-être accompagnée d'un commentaire 3 .

Cet affichage d'éléments calendaires dans l'espace public, loin d'être neutre, véhicule une dimension politique indéniable. Dans un tel contexte, je me propose d'étudier d'abord la/les réformes du calendrier romain comme marqueurs de changements politiques, à la fin du premier siècle avant notre ère. En suite de quoi je m'intéresserai beaucoup aux découpages du mois et à l'instauration de la semaine, à la nature de cette semaine et à ses enjeux politiques et religieux.

\section{Calendrier, astronomie et politique : l'année}

Les calendriers, dans toute société, sont d'abord des marqueurs politiques: leur développement et leurs changements accompagnent les transformations ou les mutations du pouvoir. Mais ce sont aussi un outil d'usage courant, voire d'usage quotidien, et il doit être compris de tous pour perdurer : il n'est que de penser à la création en 1793 du calendrier républicain dont la durée de vie n'a pas dépassé 10 ans, car, le $1^{\text {er }}$ janvier 1806, le calendrier grégorien était de nouveau en usage dans tout l'Empire.

Le lexique n'est pas neutre : les Fasti - le terme le plus utilisé pour désigner à Rome le comput officiel du temps pour une année donnée - présentent, nous l'avons vu, une connotation à la fois religieuse et juridique. Dérivant de fas dont le sens est 'permission ou ordre des dieux', 'droit divin', par opposition au ius, 'droit humain', fastus désigne de fait ce qui est autorisé par la loi divine, par l'ordre religieux. Quant à calendarium, d'où vient notre terme 'calendrier', il a originellement une dimension économique, puisqu'il désigne 'le

\footnotetext{
${ }^{1}$ Voir S. Stern, Calendars in Antiquity : empires, states and societies, Oxford, 2012, p. 169.

${ }^{2}$ Liv. IX, 46, 4-6 ; Digeste II, ii, 7.

${ }^{3}$ Macr. Les Saturnales, trad. Ch. Guittard, I 12,16 et n. ad loc.
} 
registre des dettes', dont les intérêts étaient à payer aux calendes, le premier jour du mois ; ce jour-là le pontife réunissait le peuple pour lui annoncer également les dates des jours fériés ${ }^{4}$.

\section{1/ Calendrier et travaux agricoles}

À côté du calendrier civil qui, on le verra, ne se conformait à aucun repère astronomique, il existait à Rome des calendriers agricoles nécessairement en phase - a minima - avec le mouvement (apparent) du Soleil autour de la Terre et avec les quatre saisons que ce mouvement induisait. Ce type de savoir a donné lieu, dans la littérature grecque et par suite dans la latine, à toute une série d'écrits qui, à la suite des Travaux et des Jours d'Hésiode, se sont intéressés aux travaux des champs : le texte qui a eu le plus de succès dans l'Antiquité est le poème d'Aratos de Soles (III ${ }^{\mathrm{e}}$ siècle av. J.-C.), les Phénomènes ${ }^{5}$. Loin d'être un traité d'agronomie, cette œuvre de plus de 1150 vers, qui est censée s'adresser au paysan et au marin, est constituée de deux parties : la première offre au lecteur un traité d'astronomie élémentaire et la seconde traite des signes météorologiques. Le succès de ce texte se lit dans le grand nombre de scholies - grecques ou latines $-{ }^{6}$, ainsi que dans les multiples traductions latines qui ont vu le jour de Cicéron au VIII ${ }^{\mathrm{e}}$ siècle $^{7}$ en passant par Germanicus et Aviénus ${ }^{8}$.

La forme la plus simple de ce type de calendrier était le parapegme ${ }^{9}$, mais les traités d'agronomie font état de systèmes plus complexes. P. Brind'Amour étudie deux passages du De agricultura de Caton qui lui permettent de conclure que le calendrier, adopté en 153 av. J.C. - et qui a ramené le début de l'année de mars à janvier, faisait également coïncider équinoxes et solstices avec les calendes des mois correspondants. Et d'ajouter : «Ce fut un véritable calendrier astronomique permettant la correspondance exacte des mois et des saisons [...] À aucun autre moment de son histoire, le calendrier romain n'atteignit à cette intimité de l'ordre naturel et de la convention civile ${ }^{10}$. Ainsi, pendant un temps au moins, le calendrier romain a été en coïncidence avec l'année naturelle.

\footnotetext{
${ }^{4}$ Voir Parisot, http://www.universalis-edu.com/encyclopedie/calendriers/.

${ }^{5}$ Aratos, 1998, 2 vol.

${ }^{6}$ Voir Commentariorum in Aratum reliquiae, éd. E. Maass, Berlin, 1958 ; Scholia in Aratum vetera, éd. J. Martin, Stuttgart, 1974 ; Scholia in Caesaris Germanici Aratea, éd. F. Eyssenhardt, Leipzig, 1866, éd. A. Breysig, Berlin, 1867, rééd. Hildesheim, 1967.

${ }^{7}$ L'Aratus latinus : étude sur la culture et la langue latines dans le Nord de la France au VIII siècle, éd. H. Le Bourdellès, Lille, PUL, 1985.

${ }^{8}$ Voir Bakhouche, 1996, 32-34, n. ad loc., et passim.

${ }^{9}$ Voir là-dessus RE XVIII-4, s.v. « parapegma », col. 1295-1366 [A. Rehm].

${ }^{10}$ P. Brind'Amour, 1983, 136-139 [139].
} 


\section{2/ Le calendrier civil à la fin de la République}

À l'époque républicaine, l'année comptait 355 jours, soit 29 en janvier, 28 en février, 31 en mars, 29 en avril, 31 en mai, 29 en juin, 31 en juillet, 29 en août et en septembre, 31 en octobre et 29 en novembre et en décembre. Ce calendrier n'était ni solaire ni lunaire et il manquait 10,25 jours pour que l'année romaine fût en accord avec l'année naturelle de 365 jours $1 / 4$. Pour combler ce déficit, le grand pontife décidait périodiquement de l'intercalation de 22 ou 23 jours supplémentaires. Ce 'mois' était ajouté en février, après les Terminalia du 23 et avant le Regifugium du 24, et les cinq derniers jours de février - du 24 au 28 s'ajoutaient aux jours intercalés pour former un mois intercalaire qui fût complet ; ce mois reçut le nom de mensis intercalaris et fut appelé plus tard merkedonius ${ }^{11}$, car il permettait aux créanciers de percevoir plus d'intérêts cette année-là. L'intercalation de 22 jours est présentée par Plutarque comme étant la règle générale ${ }^{12}$ et des intercalations de 22 et de 23 jours sont attestées par Censorinus (qui écrit en 238 un opuscule sur 'le jour natal', De die natali) ${ }^{13}$ et Macrobe (qui a vécu au $\mathrm{V}^{\mathrm{e}}$ siècle et, dans ses Saturnales, consacre plusieurs chapitres du livre I à l'histoire du calendrier ${ }^{14}$. Ces témoins sont unanimes à imputer aux intérêts personnels des pontifes la décision d'intercaler ou non un mois de 22 ou 23 jours :

Denique cum intercalarium mensem uiginti duum uel uiginti trium dierum alternis annis addi placuisset, ut civilis annus ad naturalem exaequaretur, in mense potissimum Februario inter Terminalia et Regifugium intercalatum est, idque diu factum, prius quam sentiretur annos ciuiles aliquanto naturalibus esse maiores. Quod delictum ut corrigeretur, pontificibus datum negotium eorumque arbitrio intercalandi ratio permissa. Sed horum plerique ob odium uel gratiam, quo quis magistratus citius abiret diutiusue fungeretur aut publici redemptor ex anni magnitudine in lucro damnoue esset, plus minusue ex libidine intercalando rem sibi ad corrigendum mandatam ultro deprauarunt... ${ }^{15}$.

${ }^{11}$ Plut. Numa 18,3 ; César 59,2.

${ }^{12}$ Numa 18,3 .

${ }^{13}$ De die natali 20,6 .

${ }^{14}$ Sat. I, 13,12.

${ }^{15}$ Cens. 20, 6-7 ; trad. G. Rocca-Serra : «Enfin, lorsqu'on eut décidé d'ajouter un mois intercalaire de vingtdeux ou vingt-trois jours une année sur deux alternativement, pour que l'année civile égalât l'année naturelle, ce mois fut intercalé de préférence en février, entre les Terminalia et le Regifugium, et cela se pratiqua pendant de longues années avant que l'on s'aperçoive que les années civiles étaient un peu plus longues que les années naturelles. On confia alors aux pontifes la charge de corriger cette erreur et permission leur fut donnée de pratiquer les intercalations selon leur jugement. Mais la plupart d'entre eux, soucieux, selon leurs haines ou leurs sympathies, de faire partir plus vite ou de conserver plus longtemps en charge un magistrat, de favoriser les gains ou de provoquer les pertes d'un percepteur des impôts par la durée plus ou moins longue de l'année, en pratiquant des intercalations arbitraires corrompirent à l'excès la matière qu'ils avaient mission de corriger... » 
Ces intercalations aléatoires avaient produit un tel décalage entre le calendrier et l'année naturelle que César, dans sa réforme du calendrier, fit passer de 355 à 445 jours - 443 selon Macrobe (Sat. I, 14,3). Écoutons encore Censorinus :

... adeo aberratum est ut C. Caesar pontifex maximus suo III et M. Aemilii Lepidi consulatu, quo retro delictum corrigeret, duos menses intercalarios dierum LXVII in mensem Novembrem et Decembrem interponeret, cum iam mense Februario dies III et XX intercalasset, faceretque eum annum dierum CCCCXLV, simul providens in futurum, ne iterum erraretur: nam intercalario mense sublato annum civilem ad solis cursum formavit. Itaque diebus CCCLV addidit decem, quos per septem menses, qui dies undetricenos habebant, ita discriberet, ut Januario et Sextili et Decembri bini accederent, ceteris singuli; eosque dies extremis partibus mensium adposuit, ne scilicet religiones sui cuiusque mensis a loco summoverentur. Quapropter nunc cum in septem mensibus dies singuli et triceni sint, quattuor tamen illi ita primitus instituti eo dinoscuntur, quod nonas habent septimanas, ceteri tres omnes alii reliqui quintanas. Praeterea pro quadrante diei, qui annum verum suppleturus videbatur, instituit, ut peracto quadrienni circuitu dies unus, ubi mensis quondam solebat, post Terminalia intercalaretur, quod nunc bis sextum vocatur ${ }^{16}$.

Les indications des charges, au début de l'extrait, permettent de dater la réforme julienne de l'an 46 av. J.-C. et Macrobe, l'autre auteur régulièrement convoqué par les historiens du calendrier, qualifie cette année d' 'ultime année de la confusion' - annus confusionis ultimus (Sat. I, 14,3). Le 'nouveau' calendrier devait être appliqué à partir de l'année suivante, en 45.

Rappelons rapidement la constitution d'un calendrier qui reste encore à ce jour le nôtre, avec le seul ajustement réalisé sous le pape Grégoire: l'année de 365 jours commence (toujours) en janvier et est constituée de 12 mois de 30 ou 31 jours ; le mois de février de 28 jours compte, tous les quatre ans, un jour de plus, pour que l'année civile coïncide avec l'année naturelle de 365 jours $1 / 4$.

\footnotetext{
${ }^{16}$ Cens. 20, 8-10 ; trad. G. Rocca-Serra : «... et il en était résulté un tel désordre que Jules César, grand pontife, consul pour la $3^{\mathrm{e}}$ fois, associé à $\mathrm{M}$. Aemilius Lepidus, désireux de corriger la faute commise dans le passé, interposa deux mois intercalaires de soixante-sept jours entre novembre et décembre, après avoir intercalé vingt-trois jours dans le mois de février, ce qui donna à cette année-là un total de 445 jours. Il prit en même temps des mesures pour qu'à l'avenir on ne tombe plus dans ces errements, et pour cela il supprima le mois intercalaire et adapta l'année civile au cours du soleil. C'est pourquoi, aux 355 jours il en ajouta dix qu'il distribua entre les sept mois de vingt-neuf jours, de manière que janvier, Sextilis et décembre aient deux jours de plus et les autres mois un jour; ces jours il les ajouta à la fin de ces mois afin que les fêtes religieuses qui y étaient célébrées ne se trouvent pas déplacées. C'est pourquoi, alors que nous avons aujourd'hui sept mois de trente-et-un jours, on reconnaît les quatre qui sont d'institution primitive à ce que les nones tombent le 7 , tandis que dans les trois autres elles tombent le 5. Enfin, pour tenir compte du quart de jour qui semblait devoir compléter l'année véritable, il ordonna qu'à la fin d'un cycle de quatre ans un jour fût intercalé à l'endroit où l'on intercalait auparavant un mois ; c'est-à-dire après les Terminalia, et que nous appelons jour 'bissexte' »; cf. Macrobe, Sat. I, 14, 6-11.
} 
À travers cette réforme du calendrier, Censorinus présente César 'désireux de corriger la faute commise dans le passé' (quo retro delictum corrigeret 20,8) ${ }^{17}$, et les historiens du calendrier ne se sont guère intéressés aux motivations du dictateur. Certes un calendrier fiable était indispensable pour un certain nombre de travaux, mais, à partir du moment où paysans et marins utilisaient d'autres outils calendaires parfaitement adaptés à leurs besoins, une nouvelle réforme du calendrier ne s'imposait pas.

\section{3/ Calendrier et pouvoir}

Le calendrier est une institution importante dans la société romaine, comme peut en témoigner la pratique, attestée à travers toute la période impériale, de graver les fastes sur des blocs de pierre ou de les peindre sur des murs, que ce soit dans des bâtiments privés ou publics, et spécialement sur les murs des temples. Il serait intéressant à ce propos de se demander si cette pratique se retrouve dans d'autres sociétés.

Il reste que la réforme julienne représente une des étapes les plus significatives de l'apparition de calendriers fixes dans l'Antiquité. Nous savons par Plutarque ${ }^{18}$ que le dictateur s'est entouré de mathématiciens, de philosophes et d'astronomes pour mener sa tâche à bien, et Pline l'Ancien précise en outre que l'appui technique au dictateur a été fourni par l'astronome alexandrin Sosigène ${ }^{19}$.

En réalité, S. Stern ${ }^{20}$ est le seul à avoir posé la bonne question des motifs de César quand il a entrepris cette vraie révolution du comput du temps. Il est fort peu vraisemblable en effet que son initiative ait eu pour finalité de faciliter les repérages temporels et météorologiques des paysans ! Les sources antiques ne nous aident pas à répondre directement à cette question, mais les divers témoignages tendent à brosser un portrait du dictateur, à dégager des éléments saillants dans la personnalité de César, qui nous permettent de comprendre la motivation du dictateur dans cette réforme. La plupart des témoins insistent sur la dimension politique de cette action. Suétone, par exemple, présente cette initiative comme la première mesure prise par César pour mettre de l'ordre dans l'État romain :

Conuersus hinc ad ordinandum rei publicae statum fastos correxit iam pridem uitio pontificum per intercalandi licentiam adeo turbatos, ut neque messium feriae aestate neque uindemiarum autumno conpeterent;

\footnotetext{
${ }^{17}$ Voir de même Suet., Caes. 40.

${ }^{18}$ Caes, 59.

${ }^{19}$ Plin. XVIII, 211. Macrobe est le seul à évoquer l'aide d'un certain 'scribe M. Flavius' (Sat. 14,2).

${ }^{20}$ Stern, 2012, 216 : « Why was the Julian calendar instituted ?»
} 
annumque ad cursum solis accommodauit, ut trecentorum sexaginta quinque dierum esset et intercalario mense sublato unus dies quarto quoque anno intercalaretur ${ }^{21}$.

Il s'agit donc d'une mesure politique d'ordre public, qui doit mettre définitivement fin à la 'confusion,22. Et Macrobe de souligner encore l'influence égyptienne qui s'est exercée sur le dictateur :

[...] imitatus Aegyptios, solos diuinarum rerum omnium conscios, ad numerum solis, qui diebus trecentis sexaginta quinque et quadrante cursum conficit, annum dirigere contendit ${ }^{23}$.

L'inspiration d'un modèle égyptien (une année de 365 jours) peut être regardée, comme le pense S. Stern (2012, p. 219), comme un élément de revendication impérialiste, ou encore comme l'expression d'une émulation avec le fameux conquérant, Alexandre le Grand, qui a subjugué les grands généraux de la fin de la République. Toujours est-il que le calendrier que César a institué - le premier calendrier fixe de l'Antiquité - était structurellement analogue à sa propre position de dictateur. La cohérence structurale entre ce nouveau mode de comput et le nouvel Empire romain sous un chef autocratique explique sans doute le choix du calendrier comme première action politique d'envergure.

En témoigne la publication immédiate de ce calendrier par édit :

Sic annum ciuilem Caesar habitis ad limam dimensionibus constitutum edicto palam posito publicauit $^{24}$.

Certes, même sans édit, le calendrier julien eût été utilisé dans l'armée, les colonies et l'administration provinciale de l'Empire romain. Mais l'édit qui impose ce nouveau comput du temps partout dans l'Empire est un geste politique fort : le calendrier constitue une élément

${ }^{21}$ Caes. 40 ; trad. H. Ailloud : «Se consacrant ensuite à la réorganisation de l'État, César réforma le calendrier, où depuis longtemps, par la faute des pontifes qui abusaient des jours intercalaires, s'était introduit un tel désordre que les fêtes des moissons ne tombaient plus en été ni celles des vendanges, en automne ; réglant l'année sur le cours du soleil, il décida qu'elle aurait trois cent soixante-cinq jours, que le mois intercalaire serait supprimé t que l'on intercalerait un jour tous les quatre ans ».

${ }_{22}$ Voir Macr., Sat. I, 14,1 (emergebat maior confusionis occasio) et 3 (annus confusionis ultimus), lexique du désordre qui s'oppose à celui d'un nouvel ordre: 2 ... C. Caesar omnem han inconstantiam temporum uagam adhuc et incertam in ordinem statae definitionis coegit...

${ }^{23}$ Sat. I, 14,3 ; trad. Ch. Guittard : « [...] à l'imitation des Égyptiens, les seuls qui aient percé tous les secrets du ciel, qui accomplit sa révolution en trois cent soixante-cinq jours et quart, qu'il s'efforça de régler la force de l'année ».

${ }^{24}$ Macr., Sat. I, 14,13 ; trad. Ch. Guittard : «César, après avoir ainsi fondé l'année civile sur des mesures prises avec la plus grande précision, la rendit publique par un édit partout affiché ». Étonnamment, D. Feeney (2007, 209-210) paraît ignorer ce passage de Macrobe, quand il insiste sur «la dimension irréductiblement romano-centrée du calendrier » 
important dans l'établissement de la Romanitas, d'une culture romaine commune qui unifie l'Empire dans une koinè culturelle sociale et politique ${ }^{25}$

Une telle interprétation est corroborée par l'institution d'une nouvelle ère, 'l'ère julienne' :

Ex hoc anno ita a Iulio Caesare ordinato ceteri ad nostram memoriam Iuliani appellantur, eique consurgunt ex quarto Caesaris consulatu ${ }^{26}$,

comme il y avait eu des ères remontant au règne d'Alexandre le Grand ${ }^{27}$, et par l'attitude similaire qu'adoptera Auguste quand il amendera à son tour le calendrier à cause d'erreurs dans les intercalations tous les quatre ans ${ }^{28}$ :

[...] cum oporteret diem qui ex quadrantibus confit quarto quoque anno confecto antequam quintus inciperet intercalare, illi [scil. sacerdotes] quarto non peracto sed incipiente intercalabant. Hic error sex et triginta annis permansit, quibus annis intercalati sunt dies duodecim cum debuerint intercalari nouem. Augustus, qui annos duodecim sine intercalari die transigi iussit, ut illi tres dies qui per annos triginta et sex uitio sacerdotalis festinationis excreuerant sequentibus annis duodecim secundum ordinationem Caesaris quinto quoque incipiente anno intercalari iussit et omnem junc ordinem aereae tabulae ad aeternam custodiam incisione mandauit ${ }^{29}$.

De fait, Censorinus, convertissant l'année où il écrit son opuscule De die natali selon les différentes ères romaines, donne les précisions suivantes :

Secundum quam rationem nisi fallor hic annus, cuius uelut index et titulus quidam est V. C. Pii et Pontiani consulatus, ab Olympiade prima millensimus est et quartus decimus, ex diebus dumtaxat aestiuis, quibus agon Olympicus celebratur; a Roma autem condita nongentesimus nonagensimus primus, et quidem ex Parilibus, unde urbis anni numerantur; eorum uero annorum, quibus Julianis nomen est, ducentesimus octogensimus

${ }^{25}$ Stern, 2012, 226 ; pour tout ce paragraphe, voir ibid., 211-227.

${ }^{26}$ Cens. 20,11 ; trad. G. Rocca-Serra : « À partir de l'année ainsi organisée par Jules César, les années suivantes jusqu'à notre époque sont appelées 'juliennes' et prennent leur départ du $4^{e}$ consulta de César ». Voir Grumel, 1958, 208-209.

${ }^{27}$ Grumel, 1958, 209.

${ }^{28}$ Voir là-dessus les témoignages Beaujeu, 1975.

${ }^{29}$ Macr., Sat. I, 14,14-15 ; trad. Ch. Guittard : « [...] alors qu'ils auraient dû intercaler les jours résultant des quarts de jour, tous les quatre ans révolus avant le début de la cinquième année, ceux-ci [scil. les prêtres] l'intercalaient non pas à la fin mais au commencement de la quatrième année. Cette erreur persista trente-six ans, au cours desquels furent intercalés douze jours, quand neuf seulement auraient dû l'être. Mais on finit aussi par découvrir cette erreur qui fut corrigée par Auguste, lequel ordonna de laisser passer douze années sans jour intercalaire pour que l'excédent des trois jours survenu à cause de la trop grande hâte des pontifes pendant trente-six ans fût absorbée par l'absence de jour intercalaire au cours des douze années suivantes. Après quoi, il ordonna d'intercaler un jour au début de chaque cinquième année, conformément aux dispositions de César, et il fit graver sur une table d'airain l'ensemble de ces mesures, pour qu'elles fussent éternellement observées. » 
tertius, sed ex die kal. Januariarum, unde Iulius Caesar anni a se constituti fecit principium; at eorum, qui uocantur anni Augustorum, ducentesimus sexagensimus quintus, perinde ex kal. Januariis [.... $]^{30}$.

Pour éphémères qu'elles aient été, la création des ères 'julienne' et 'augustéenne' souligne la réussite des ces nouveaux maîtres de Rome qui n'ont pas simplement marqué leur époque, mais l'ont révolutionnée, en particulier en donnant leur marque au comput du temps.

\section{Les divisions traditionnelles du mois}

Les historiens du calendrier s'intéressent essentiellement à la macro-structure calendaire, c'est-à-dire au découpage de l'année en mois, mais les divisions du mois n'intéressent guère la critique. Pourtant la substitution de la semaine aux divisions mensuelles constitue, à Rome, une autre révolution qui reste peu et mal appréhendée. En témoignent les deux textes utilisés jusqu'ici comme références - celui de Censorinus au III ${ }^{\mathrm{e}}$ siècle (qui passe de l'année au jour) et de Macrobe au $\mathrm{V}^{\mathrm{e}}$ siècle - qui ne parlent absolument pas des découpages du mois.

\section{1/ L'usage ancestral}

Le mois romain archaïque présentait déjà des particularités qui ont perduré : sa division en trois parties inégales, l'inscription dans le calendrier des jours Fastes, Néfastes et de nundinae, des marchés tous les huit jours ; mais surtout le comput des jours se faisait 'à reculons' à partir de la division suivante.

Le calendrier préjulien était en effet un calendrier originellement lunaire qui comptait 355 jours, soit 12 mois de 29 jours et demi en moyenne. Le mois commençait le jour des calendes, était coupé en son milieu par les ides (le 13 ou le 15 selon les mois) et en son premier quart par les nones (le 5 ou le 7). Le nom même de nones qui vient de nonus, 'neuvième', instituait ce jour en neuvième position avant les ides, c'est-à-dire huit jours avant.

${ }^{30}$ Cens. 21,6-8 ; trad. G. Rocca-Serra : En me conformant à ses calculs [scil. ceux de Varron] et si je ne me trompe pas, l'année où nous sommes, et dont le consulat des clarissimes Pius et Pontanus constitue comme la marque distinctive ou le titre, est la $1014^{\mathrm{e}}$ à partir de la première Olympiade, et précisément à partir de l'été, époque où se célèbrent les jeux. À partir de la fondation de Rome, c'est la $991^{\mathrm{e}}$ année, en prenant pour point de départ les Parilia, d'où sont comptées les années de Rome ; en années juliennes, c'est la $283^{\mathrm{e}}$ à partir des calendes de janvier, dont Jules César a fait le commencement de l'année qu'il avait constituée ; en années augustéennes, c'est la $265^{\mathrm{e}}$ à partir des calendes de janvier [...]». 
Lexicalement, les 'calendes' viennent du verbe grec calo, 'appeler, proclamer', selon Varron qui donne également, dans son De Lingua Latina, l'étymologie des deux autres termes :

Primi dies mensium nominati Kalendae, quod his diebus calantur eius mensis Nonae a pontificibus, quintanae an septimanae sint futurae, in Capitolio in Curia Calabra sic dicto quinquies: 'calo, Iuno Couella', septies dicto : 'calo, Iuno Couella'.

Nonae appellatae aut quod ante diem nonum Idus semper aut quod, ut nouus annus Kalendae Ianuariae ab nouo sole appellatae, nouns mensis <ab> noua luna Nonae [...] Idus ab eo quod Tusci itus, uel potius quod Sabini edus dicunt ${ }^{31}$.

Quant aux nundinae, Varron écrit ailleurs :

Itaque annum ita diuiserunt ut nonis modo diebus urbanas res usurparent, reliquis septem ut rura colerent $^{32}$.

Contrairement à ce qu'a pu suggérer J. Heurgon, il ne s'agit pas là de « la semaine classique de huit jours, signalée dans les Fastes par les huit premières lettres de l'alphabet $»^{33}$, mais bien de ce que signalent par là les calendriers, à savoir des jours de marchés dont la périodicité est tous les neuvièmes jours.

Dans une perspective purement astronomique, les calendes correspondent, dans un calendrier strictement lunaire, à la Nouvelle Lune et les ides à la Pleine Lune. Toujours est-il que la plus grande difficulté à manipuler le comput des jours à Rome était moins d'ordre astronomique, pour les faire coïncider à une position remarquable de la Lune, que d'ordre pratique pour trouver le numéro d'ordre du jour à partir des nones, ides ou calendes du mois suivant. C'est peut-être ce qui explique les multiplications d'affichage de calendriers dans

${ }^{31}$ LL VI, 4, 27-28 ; trad. P. Flobert : «Le premier jour des mois a été appelé Calendes, parce que c'est le jour où les Nones de ce mois, selon qu'elles doivent être le cinq ou le sept, sont proclamées (calantur) par les pontifes, sur le Capitole dans la curia Calabra ; ainsi il est dit cinq fois : 'je proclame, Junon Covella', il est dit sept fois : 'je proclame, Junon Covella'.

Les Nones portent ce nom soit parce qu'elles tombent toujours le neuvième jour avant les ides, soit parce que, au même titre que les Calendes de janvier sont appelées 'nouvel an' à cause du soleil nouveau, les Nones sont appelées 'nouveau mois' à cause de la nouvelle lune [...]

Les Ides viennent de ce que les Étrusques disent itus ou plutôt que les Sabins disent edus ». Voir notes ad loc.

${ }^{32} R R$ II, 1 ; trad. Ch. Guiraud : «C'est pourquoi ils divisèrent l'année de manière à ne traiter les affaires de la ville qu'un jour sur huit et à cultiver les champs pendant les sept jours qui restaient ».

${ }_{33} 1948$, 241. Voir cependant infra «Un rythme hebdomadaire ». 
l'Empire romain, comme les Fasti Antiates reproduit en annexe à la fin de cette étude ${ }^{34}$. C'est le plus ancien des calendriers romains et l'unique calendrier pré-julien qui nous soit parvenu ; il est représenté sous forme d'un tableau à treize colonnes. Chaque colonne concerne un mois dont le nom est abrégé comme suit: IAN pour Ianuarius (janvier), FEB pour Februarius (février), MAR pour Martius (mars), APR pour Aprilis (avril), MAI pour Maius (mai), IVN pour Innius (juin), QVI pour Quintilis (juillet), SEX pour Sextilis (août), SEP pour September (septembre), OCT pour October (octobre), NOV pour November (novembre), DEC pour December (décembre) et INTER pour Intercalaris (mois intercalaire).

Chaque colonne comporte verticalement autant de lignes que le mois comporte de jours, plus une ligne supplémentaire où est indiqué le nombre de jours que comporte le mois : XXXI (trente-et-un) pour les mois de mars, mai, juillet et octobre ; XXIX (vingt-neuf) pour les mois de janvier, avril, juin, août, septembre, novembre et décembre ; XXIIX (vingt-huit) pour le mois de février ; XXVII (vingt-sept) pour le mois intercalaire. Le premier jour de chaque mois est marqué de la lettre $\mathrm{K}$, abréviation de kalendae (calendes). Le cinquième jour des mois de janvier, février, avril, juin, août, septembre, novembre et décembre est marqué des lettres NON, abréviation de nonae (nones) ; il en est de même du septième jour pour les autres mois (mars, mai, juillet et octobre). Le huitième jour suivant les nones de chaque mois est marqué par les lettres EI pour eidus/idus (ides).

Certains jours enfin sont affectés d'une des trois lettres suivantes : F, N ou C. La lettre F, abréviation de fastus (dies), marque les jours fastes ; la lettre $\mathrm{N}$, abréviation de nefastus (dies), les jours néfastes ; la lettre C, abréviation de comitiales (dies), les jours 'comitiaux', c'est-àdire les jours pendant lesquels les comices peuvent être convoqués.

Dans un autre témoignage archéologique, les Fasti Verolani, qui offre un calendrier julien, aux indications déjà trouvées s'ajoutent des indications de fêtes mais aussi un outil indispensable pour l'usager: la position numérique des jours par rapport à la division suivante : ainsi le 6 février (voir EviIIN) est le $8^{\mathrm{e}}$ jour avant les ides de février et le $12 \mathrm{du}$ même mois (CPRN) la veille des ides.

On a là deux beaux exemples des renseignements attendus - et trouvés - dans ces types d'inscription.

\section{2/ Un rythme hebdomadaire}

\footnotetext{
${ }^{34}$ Dessin $n^{\circ} 2$.
} 
Il est bien évident que dans les traités d'agronomie, c'est la Lune qui rythme les différents travaux agricoles ; c'est elle que doit observer le paysan pour accomplir des tâches tributaires des saisons et conditionnées soit par la lune croissante soit par la lune décroissante. Rappelons que ce sont des repères célestes qu'observent les Anciens pour se situer dans l'année naturelle ; or ces repères - lever ou coucher de telle constellation, âge de la Lune - ne sont visibles que la nuit.

Chacun des trois livres de l'Économie rurale de Varron, ouvrage dédié à son épouse Fundania, se présentent sous forme de trois dialogues supposés avoir eu lieu à des dates et dans des circonstances différentes. Au c. 2 du livre I, Varron dit avoir rencontré son beaupère, Fundanius, C. Agrius, «chevalier romain et philosophe socratique » et P. Agrasius, «publicain ». La conversation s'engage entre eux quand ils voient arriver C. Licinius Stolon et Cn. Tremelius Scrofa. En I, 37, Agrius fait allusion à une division en quatre parties du mois lunaire sur laquelle il souhaite avoir l'avis de ses amis. Et Scrofa de répondre :

'Numquam rure audisti', inquit, 'octauo Ianam Lunam et crescentem et contra senescentem, et quae crescente luna fieri oporteret, [et] tamen quaedam melius fieri post octauo Ianam lunam quam ante? Et siquae senescente fieri conueniret, melius, quanto minus haberet ignis id astrum? Dixi de quadripertita forma culturae agri ${ }^{35}$.

Déjà Caton avait évoqué ce type de division :

Per hiemem lucubratione haec facito: ridicas et palos, quos pridie in tecto posueris, siccos dolato, faculas facito, stercus egerito. Nisi intermestri lunaque dimidiata tum ne tangas materiem. Quam effodies aut praecides abs terra, diebus VII proximis, quibus luna plena fuerit, optime eximetur ${ }^{36}$.

J. Heurgon rappelle que ce petit texte a été abondamment utilisé par les successeurs de Caton : Varron s'en est souvenu (RR I, 37,1); Columelle en donne une paraphrase (RR XI, 2,11) et Pline le cite deux fois et, la seconde textuellement (XVI 190 et 194). L'expression

\footnotetext{
${ }^{35} R R$ I, 37 ; trad. J. Heurgon légèrement modifiée : «'N'as-tu jamais entendu parler à la campagne', dit-il, 'du huitième jour avant Notre-Dame la Lune, pleine ou nouvelle ? Parmi les choses qu'il fallait faire pendant le croissant de la lune, il y en avait pourtant qu'il valait mieux faire après le huitième jour avant Notre-Dame la Lune qu'avant. Quant à celles qu'il convenait de faire dans son déclin, mieux valait que cet astre eût moins de feu. Voilà ce que j'avais à dire de la forme quadripartite en agriculture' ».

${ }^{36}$ Agr. 37(XLII, 3-4) ; trad. R. Goujard : «Pendant 1'hiver, à la chandelle, faites ces travaux : façonnez à la dolabre, une fois secs, les échalas et les piquets que vous aurez mis la veille à couvert, faites des torches, sortez le fumier. Si ce n'est à l'interlune, et quand la lune est réduite de moitié, ne touchez pas alors au bois d'œuvre ; celui que vous arracherez ou que vous couperez sur pied, dans les sept jours qui suivront la pleine lune, sera exploité dans les meilleures conditions ».
} 
diebus VII proximis, quibus luna plena fuerit semble signifier : «sept jours après la pleine lune », et le langage de Caton est clair : sept jours après la pleine lune, l'astre de la nuit entre dans son dernier quartier. On a là une semaine de sept jours bien délimitée, qui s'insère entre la semaine de sept jours qui précède la pleine lune (octauo Ianam Lunam crescentem) et celle qui précède la nouvelle lune '(octauo Ianam Lunam decrescentem $)^{37}$.

Cet usage de la quadripartition du mois lunaire s'impose tout naturellement dans les textes d'agronomie.

Un peu après l'époque de Varron, Hygin, le bibliothécaire de l'empereur Auguste, évoque, lui, une division du moins en huit parties. Nous sommes au début du livre IV de son Astronomie, qui traite des cercles célestes, et notre auteur essaie de prouver que les cercles sont divisés en 8 parties et pas 12 ou en n'importe quel autre nombre : non dubium est quin recte diuidatur in partes octo («donc la bonne règle est la division en huit parties »). Il illustre cette conclusion par cinq preuves, usant chaque fois de l'anaphore de l'adverbe praeterea pour les introduire, et voici la dernière et cinquième de ces preuves :

Praeterea cum uideamus octauo quoque die eiusdem sideris horam primam, aut quamlibet denique esse, et cuius hodie fuerit hora prima, eiusdem octauam quoque futuram, uerissimum est ipsam quoque sphaeram mundi, unde horae sumuntur, diuidi in partes VIII $^{38}$.

Contrairement à ce que pense A. Le Boeuffle ne joue pas sur la double valeur possible de l'ordinal dans la formule octauo quoque die : le sens littéral est bien « chaque huitième jour », c'est-à-dire tous les sept jours révolus ${ }^{39}$.

\section{La semaine : la seconde révolution}

La semaine comme division du mois paraît s'être lentement substituée aux divisions traditionnelles en calendes, nones et ides. Son nom latin septimana, qui apparaît dans les

\footnotetext{
${ }^{37}$ Voir Heurgon, 1948, 245, qui présente le latin entre parenthèses en ordre inversé ; mais la Lune, avant PL, est en mouvement croissant et, dans la seconde partie du mois, en mouvement décroissant.

${ }^{38}$ Astr. IV , 2,2 ; trad. A. Le Boeuffle modifiée : «De plus, nous voyons tous les sept jours la première heure ou toute autre appartenir au même astre et celui qui est aujourd'hui maître de la première heure le sera aussi de la huitième ; il est donc tout à fait conforme à la vérité qu'à son tour, la sphère céleste aussi, origine des heures, soit divisée en huit parties $\gg$.

${ }^{39}$ Astr., p. 205 n. 13.
} 
textes chrétiens ${ }^{40}$ avant de figurer dans le code Théodosien $(15,5,5)$, indique sa relation essentielle au nombre 7 et ne saurait correspondre à la périodicité des nundinae, comme certains l'ont dit ${ }^{41}$.

Quand le Nicéen Dion Cassius ( $\approx 150$-après 235 ) écrit son Histoire romaine, la semaine est en usage dans l'Empire. C'est au livre 37 que l'historien, évoquant le siège et la prise de Jérusalem par les troupes romaines commandées par Pompée, consacre un excursus de plusieurs chapitres sur les juifs, en lien avec leur respect du 'jour de Saturne', jour au cours duquel ils ne se livrent à aucune activité. À quoi Dion ajoute, de but en blanc, deux chapitres sur le principe d'un comput septénaire associé à celui des jours ; mais surtout il témoigne de l'utilisation généralisée de la semaine dans tout l'Empire. Ce texte est régulièrement exploité par les historiens du calendrier.

\section{1/ L'évolution des mentalités et l'importance du 7}

Dion Cassius ramène le principe de la semaine de sept jours à deux systèmes $(\S 18)$ : le premier ressortit à l'harmonie musicale et le second aux patronages astraux de chaque heure du jour.

Le texte de Dion sur les intervalles musicaux entre les astres est assez abscons, car il paraît mélanger le principe de l'harmonie musicale d'origine pythagoricienne et celui des protections astrales, ramené aux égyptiens par Dion, de chaque heure et par suite de chaque jour. Dans le système musical, il s'agit de 'sauter' deux planètes. On passe ainsi de Saturne (= samedi) au Soleil (= dimanche) et du Soleil à la Lune (= lundi); si l'on continue à sauter de deux en deux à partir de la Lune, on arrive à Mars (= mardi) puis à Mercure (= mercredi) et ainsi de suite. Ce qui fait problème ici c'est l'absence de toute référence musicale propre, qu'il s'agisse d'intervalles ou d'octave. À l'origine, dans la tradition gréco-latine, les développements musicaux sont convoqués pour expliquer la partition de l'âme du monde par le démiurge dans le Timée de Platon (35B-36D). Il est question en effet de diviser la substance de l'âme sept fois de la façon suivante : «[le démiurge] retrancha une seule part sue le tout [1] ; après celle-ci, il en retrancha une seconde, double de la première [2] ; et encore une troisième qui, valant une fois et demie la seconde, était le triple de la première [3]; une quatrième double de la seconde [4]; une cinquième triple de la troisième [9]; une sixième valant huit fois la première [8]; et une septième valant vingt-sept fois la première [27]».

\footnotetext{
${ }^{40}$ Ps-Cypr. Pasch.comp. 17, 18 ; Aug. Serm. 268,1; Cassian. Inst. 3, 9, 3 et passim ; Hier. Ier. 2, 6, 22, etc.

${ }^{41}$ Voir Colson, 1926, 3-5.
} 
L'écart entre ces nombres a été comblé par des fractions numériques correspondant à des intervalles musicaux : 4/3 à la quarte, $3 / 2$ à la quinte et $9 / 8$ au ton, le produit de la quarte par la quinte donnant 2, qui correspond à l'octave.

Cette construction mathématico-musicale était bien connue de tous les lettrés à Rome, dès l'époque classique. Cicéron, au livre VI de son De republica, met en scène Scipion Émilien qui, le temps d'un songe, se trouve transporté dans l'espace d'immortalité que constitue la Voie Lactée (là encore une idée pythagoricienne). Il y rencontre son père et son grand-père qui lui révèlent les secrets de l'univers et son propre destin à lui, le 'jeune' Scipion, qui mourra âgé de 8 fois 7 ans. Plusieurs siècles plus tard, Macrobe commentera ce songe en mettant en avant la dimension pythagoricienne de toute cette fiction.

Varron, contemporain de Cicéron et néopythagoricien avéré, aurait développé les idées du Maître dans des ouvrages aujourd'hui perdus, mais dans son Économie rurale, la longue description, en III, 5 , de sa volière révèle la dimension pythagoricienne de la construction ${ }^{42}$. Censorinus également, dans son De die natali, consacre un long développement aux vertus du nombre 7 (c. 11) et consacre un petit développement - fort clair - à cette fameuse musique des sphères célestes :

Ad haec accedit quod Pythagoras prodidit hunc totum mundum musica factum ratione, septemque stellas inter caelum et terram uagas, quae mortalium geneses moderantur, motum habere enrythmon et interualla musicis diastematis congrua, sonitusque uarios reddere pro sua quaeque altitudine ita concordes, ut dulcissimam quidem concinant melodian, sed nobis inaudibilem propter uocis magnitudinem, quam capere aurium nostrarum angustiae non possint ${ }^{43}$.

Les chrétiens à leur tour adoptent partie de ces idées, soit sous l'influence de leur milieu culturel, soit sous celle des juifs. C'est ainsi que Jésus choisit douze apôtres parce que le peuple d'Israël à l'origine était composé de douze tribus, chiffre lui-même symbolique : il indique par ce chiffre le nouveau peuple de Dieu. Les auteurs du Nouveau Testament ont tout naturellement puisé dans la symbolique de l'Ancien Testament, bien connue de leurs lecteurs, pour mettre en lumière le mystère du Christ. Dans l'évangile selon saint Marc figurent deux

\footnotetext{
${ }^{42}$ Voir Des Anges \& Seure, 1932 ; Deschamps, 1987 ; Sauron, 1994 ***; Étienne, 2006.

${ }^{43}$ Cens. 13,1 ; trad. G. Rocca-Serra : «En outre, Pythagore a affirmé que tout notre univers était constitué à partir d'une ordonnance musicale, que les sept planètes qui errent entre le ciel et la terre et qui commandent à la génération des mortels, ont un mouvement harmonique et des distances respectives correspondant à des intervalles musicaux; elles émettent des sons différents en proportion de leur hauteur, accordés de telle sorte qu'il en résulte la plus agréable des mélodies, mais que nous ne pouvons pas entendre du fait de la grandeur des sons, à cause de la faiblesse de nos oreilles à la saisir ». Voir la référence aux vertus stellaires du nombre 7 extraite du livre I des Hebdomades de Varron ap. Gell. III, 10, 6.
} 
récits de multiplication des pains : à la fin du premier (Mc 6, 30-44), il reste douze corbeilles pleines, le nombre des douze tribus ; dans le second récit (Mc 8, 1-10), il reste sept corbeilles, ce nombre évoquant la complétude selon Dieu. On pourrait multiplier les exemples.

Pour Charles Piétri, dans son étude «Le temps de la semaine à Rome et dans l'Italie chrétienne (IV-VI ${ }^{\mathrm{e}}$ s.) ${ }^{44}$, les chrétiens reprennent des éléments de la cosmologie juive, par exemple le gouvernement des sept planètes par sept archanges; «elle distribuait les hiérarchies angéliques dans l'échelle cosmique des sept cieux en transposant dans l'espace céleste la progression septénaire de l'hebdomade temporelle ».

En revanche, les chrétiens ont souvent tenté de s'opposer à la dimension astrologique de la semaine astrale qui a, selon Dion, prévalu dans tout l'Empire romain :

«Si l'on énumère les heures du jour et de la nuit, en commençant par la première, en l'attribuant à Cronos, la suivante à) Zeus, la troisième à Arès, la quatrième au Soleil, la cinquième à Aphrodite, la sixième à Hermès, la septième à la Lune, selon l'ordre des orbites établi par les Égyptiens, si l'on répète l'opération, en parcourant ainsi l'ensemble des vingt-quatre heures, on constatera que la première heure du jour suivant fait revenir au Soleil ; si l'on étend l'opération aux vingt-quatre heures selon le même processus que pour les autres auparavant, on assignera la première heure du troisième jour à la Lune, et si l'on procède ainsi de l'une à l'autre de celles qui restent, chaque jour recevra la divinité qui lui convient ${ }^{45}$.

Ce système peut se présenter ainsi, en commençant par Saturne :

$\begin{array}{ll}\text { Saturne } & 1^{\text {ère }} \text { heure : Saturne } \\ & 2^{\mathrm{e}} \text { heure : Jupiter } \\ & 3^{\mathrm{e}} \text { heure : Mars } \\ & 4^{\mathrm{e}} \text { heure : Soleil } \\ & 5^{\mathrm{e}} \text { heure : Vénus } \\ & 6^{\mathrm{e}} \text { heure : Mercure } \\ & 7^{\mathrm{e}} \text { heure : Lune } \\ & 8^{\mathrm{e}} \text { heure : Saturne } \\ & 9^{\mathrm{e}} \text { heure : Jupiter } \\ & \ldots \\ & 23^{\mathrm{e}} \text { heure : Jupiter } \\ & 24^{\mathrm{e}} \text { heure : Mars }\end{array}$

${ }^{44} 1984,67$.

${ }^{45}$ Dion Cassius XXXVII, 19, trad. M. Bonnefond-Coudry. 


$$
\begin{array}{ll}
2^{\mathrm{e}} \text { jour } & 1^{\text {ère }} \text { heure : Soleil } \\
& 2^{\mathrm{e}} \text { heure : Vénus etc. }
\end{array}
$$

\section{2/ Le rythme hebdomadaire du temps : une révolution dans le comput}

À la différence du premier système de la semaine, de nature harmonique, qui était seulement astral, le second est nettement marqué par l'astrologie : il s'agit de la protection, évoquée déjà par Hygin, de chaque heure d'une journée par une planète. Les 24 heures du jour offrant 3 cycles de 7 (c'est le nombre des planètes) plus 3, le premier jour, Saturne, est placé sous la protection de cette planète-dieu parce que sa première heure est sous ce même patronage. Le jour suivant est placé sous le signe de la troisième planète après Saturne, soit le Soleil, Solis dies, et ainsi de suite jusqu'à épuisement des 168 heures de la semaine.

Dion ramène à juste titre la semaine aux Égyptiens : leur calendrier cependant, à l'origine, ne connaissait pas la semaine, mais offrait une division ternaire des mois qui correspondait aux décans en astrologie, soit trois décades comme la division d'un signe zodiacal de $30^{\circ}$ se divisait en trois décans de $10^{\circ}$ chacun. Ce détail suffit cependant à souligner les interactions entre calendriers.

Une telle 'internationalisation' des marqueurs temporels est déjà une première, témoignant de la plasticité des outils de comput du temps à partir de la réforme julienne.

Une seconde révolution ressortit, à Rome, au nouveau comput des jours dans le mois et dans l'année :

- l'année et le mois ne commencent plus par le même type de jour (calendes),

- le comput ne se fait pas 'à reculons' mais dans le sens direct d'écoulement du temps,

- la fin de la semaine ne coïncide que très rarement avec la fin du moins ou de l'année.

Dès lors les Fasti affichés ne valent désormais que pour une année, comme nos calendriers modernes ${ }^{46}$.

3/ L'apparition de la semaine : status quaestionis

\footnotetext{
${ }^{46}$ Parmi les Fasti conservés, voir celui de 354 étudié par Stern, 1953.
} 
La question de l'apparition de la semaine dans le monde romain est une quaestio uexata. Si le texte de Dion peut constituer un terminus post quem, les avis des spécialistes varient pour le terminus a quo. Ils relèvent le plus souvent de l'intime conviction, sans la moindre preuve. Pour Alan E. Samuel, « [Weeks] are not attested until quite late in Greek or Latin sources, and there is no doubt that the tradition of the week finds its origin in Hebrew pratice ${ }^{47}$. De la même manière, Jörg Rüpke écrit : «As early as the first century BCE, the eight-day week was joined in Rome by a week of seven days, originating in Babylonia [...] The Babylonian week reached Rome by two routes, one of them in the form of the Jewish Sabbath observed as a rest day, and the other via the Greek planetary week... $»^{48}$. Pierre Brind'amour, arguant d'une allusion au jour de Saturne dans un passage de Tibulle (I, 3, 15-18), conclut abusivement que «le texte nous renseigne [...] sur la dissémination de la semaine en dehors des milieux juifs $»^{49}$. Pour Charles Piétri, l'«interprétation planétaire du sabbat gagne jusqu'au mouvances plus ou moins christianisées du judaïsme [...] Tacite confond sabbat et dies Saturni parce que l'Italie impériale connaît le développement parallèle d'une semaine planétaire », et plus loin : «Une chose paraît sûre : la diffusion d'une nomenclature, pour désigner les sept jours d'un cycle dont les juifs ont tracé, avec une conception du temps, un modèle original $»^{50}$. Pour A. Klaas Worp enfin, le terminus a quo est très tardif : «The earliest attestations of such weekday indications in both eht 'Eastern' and 'Western' documents come from the early third century A.D. $»^{51}$.

Les premières attestations sont en effet rares. Elles sont de deux sortes, soit littéraires, soit épigraphiques. Ce qui peut apparaître comme la plus ancienne référence à la semaine astrale se lit dans le Satiricon de Pétrone, qui aurait été écrit sous Néron, avant la conjuration de Pison $^{52}$. Au chapitre 30, Encolpe et ses amis, invités au banquet de Trimalcion, découvrent, à la porte du triclinium, deux tableaux attachés aux battants de la porte :

471972,18 .

482011,162 .

$491983,259$.

501984,67 et 70 .

${ }^{51} 1991,221$.

${ }^{52}$ Voir Petr., 1923, introduction p. XII ; cf. de même Martin, 1999, 7-8, et Puccini-Delbey qui conclut à un non liquet : « La question de la datation reste encore ouverte à l'heure actuelle » (2010,91-92). 
[...] et duae tabulae in utroque poste defixae, quarum altera, si bene memini, hoc habebat inscriptum : 'III. et pridie kalendas Ianuarias C. noster foras cenat', altera lunae cursum stellarumque septem imagines pictas; et qui dies boni quique incommodi essent, distinguente bulla notabantur ${ }^{53}$.

Cette ekphrasis paraît décrire un calendrier sur lequel les images des planètes représentent les jours, un peu comme dans le parapegme des thermes de Trajan, plus tard.

Datant sensiblement de la même période, et précisément de 60 de notre ère, un graffito (CIL IV 4182 ${ }^{54}$ ) découvert à Pompéi se présente de la façon suivante :

\author{
Nerone Caesare Augusto \\ Cosso Lentulo Cossi fil. cos \\ VIII Idus febrarais \\ dies solis, luna XIIIIX, nun(dinis) Cumis, V nun(dinas) Pompei
}

Selon cette inscription, le 6 février de l'an 60 de notre ère aurait été un dimanche, le $16^{\mathrm{e}}$ jour de la Lune et le jour de marché se tenait à Cumes et à cinq jours du marché de Pompéi. Sans entrer dans les difficultés de l'inscription car cette date très précise correspondrait à un mercredi et non à un dimanche ${ }^{55}$, la datation de l'inscription se fait en deux temps : la référence aux ides permet de donner la date, le tantième du mois, et l'allusion au Soleil indique le jour de la semaine qui correspond à cette date. On a donc un double mouvement de lecture calendaire : dans le sens direct avec les jours de la semaine et 'à reculons' pour la datation traditionnelle.

Un autre graffito, trouvé également à Pompéi (reg. III, ins. IV), offre un index des nundinae : se présentant sous huit colonnes, le document donne, en col. 1, la liste des jours (sous dies) du samedi au vendredi suivant; la col. 2 donne les lieux des marchés et porte en titre nundinae; les col. 3, 4 et 5 donnent les jours du mois du 13 ( ?) octobre au 12 novembre, et les trois dernières (de 6 à 8 ) comptent les jours d'un mois de 30 jours, du $1^{\text {er }}$ au 30 , sans les découpages traditionnels en calendes, nones et ides ${ }^{56}$.

${ }^{53}$ Satir. 30, 3 ; trad. A. Ernout : « [...] et sur les battants de la porte étaient attachés deux tableaux. L'un d'eux, si j'ai bonne mémoire, portait cette phrase : 'L'avant-veille et la veille des calendes de janvier notre maître Caius dîne en ville' ; sur l'autre étaient peints le cours de la lune, et les figures des sept planètes ; les jours favorables et les jours néfastes y étaient en outre notés par des boules de couleurs différentes ».

${ }^{54}$ Mau \& Zangmeister, 1898, I, 2, 515 = Degrassi, 1963, 330.

${ }^{55}$ Ibid., et voir Brind'amour, 1983, 268-269.

${ }^{56}$ Voir Mau \& Zangmeister, 1898, IV, 3,1, 921, et dessin $n^{\circ} 2$. 
Enfin les auteurs chrétiens utilisent un comput des jours hérité du judaïsme où seul le nom de chabbat, 'le septième' (de cheva, 'sept'), a été transmis sous cette appellation, tandis que les autres jours de la semaine portent simplement un numéro d'ordre.

Tertullien (160-220), dans son traité Sur le jeûne écrit en 201, écrit :

[...] sic et apostolos obseruasse, nullum aliud imponentes iugum certorum et in commune omnibus obeundorum ieiuniorum, proinde nec stationum, quae et ipsae suos quidem dies habeant quartae feriae et sextae, passiue tamen currant $[\ldots]^{57}$.

Le passage est intéressant par la différence sans doute délibérée entre les pratiques des chrétiens et celles des nations : il convient de signaler l'hapax sémantique de feria comme synonyme de dies, alors que le mot, généralement utilisé au pluriel en latin classique, désignait les fêtes, les jours fériés. Il nous renseigne également sur le point de départ du comput des jours ; cette phrase permet de remonter au premier jour de la semaine : le sixième jour en effet correspondant au vendredi (jour de jeûne en effet dans le christianisme antique) et le quatrième, par suite, au mardi, la semaine commençait le dimanche.

Un peu plus loin dans le même traité, c'est le mot sabbati, étrange translittération de l'hébreu chabbat, qui est utilisé comme synonyme de feria :

[...] Cur quinquaginta exinde diebus in omni exultatione decurrimus? Cur stationibus quartam et sextam sabbati dicamus et ieiuniis parasceuen $?^{58}$

Là encore il est question des deux jours de jeûnes de la semaine, le mardi et le vendredi, dans une terminologie déjà présente dans les évangiles ${ }^{59}$.

Cette fidélité de Tertullien - et des évangélistes - à la tradition juive dans le comput des jours contredit la remarque de Max Lejbowicz dans son étude sur les tables pascales :

«En s'institutionnalisant, le christianisme réunit des traditions culturelles d'origine diverse sans manifester le moindre embarras $»^{60}$.

À ce stade du développement de la nouvelle religion, on constate au contraire une fidélité au comput de l'Ancien Testament et à la semaine hébraïque, et par là même un refus général de la semaine astrale vue comme ce qu'elle était : un découpage astrologique ${ }^{61}$.

\footnotetext{
${ }^{57}$ Ieiu. 2, 3 ; même utilisation du mot feria chez Irénée V, 3,2.

${ }^{58}$ Iеiu. 14,2 .

${ }^{59}$ Luc. 24,1 ; Mat. 16,2 ; Io. 20,1.

${ }^{60}$ Lejbowicz, http://methodos.revues.org/575?file $=1,5$.

${ }^{61}$ Voir Pietri, 1984, 73.
} 


\section{4/ Réactions et résistances}

La diversité des dénominations des jours de la semaine en milieu chrétien et la double datation - par les découpages traditionnels du mois et par les jours de la semaine - ne suppriment manifestement pas l'usage traditionnel du comput des jours par référence aux calendes, nones et ides.

Dans une période tardive, au $\mathrm{V}^{\mathrm{e}}$ siècle, soit un siècle après le décret de Constantin qui avait ordonné à la plèbe des villes de chômer «le vénérable jour du Soleil, uenerabili die Solis ${ }^{62}$, Macrobe évoque le partage du mois selon les trois repères 'antiques' et ne paraît pas connaître d'autres divisions du mois :

[...] Sic factum est ut alii triginta et unum alii undetriginta sortirentur dies. Omnibus tamen mensibus ex die Nonarum Idus nono die repraesentari placuit : et inter Idus ac sequentes Kalendas constitutum est sedecim dies esse numerandos. Ideo mensis uberior duos illos quibus augebatur dies inter Kalendas suas et Nonas habebat. Hinc aliis quintus a Kalendis dies aliis septimus Nonas facit. Caesar tamen [...] stata sacra custodiens, nec in illis mensibus quibus binos adiecit dies ordinem uoluit mutare Nonarum, quia peractis totius mensis feriis dies suos rei diuinae cautus inseruit ${ }^{63}$.

Il est vrai qu'il s'agit d'abord du calendrier de Romulus dont nous avons dans cet extrait la conclusion avant la référence au calendrier de César. L'extrait ne fait sans doute pas apparaître à quel point le passage relatif au calendrier primitif abonde en termes qui insistent sur le hasard et les méthodes empiriques, comme l'indique d'ailleurs, dans la première phrase, le choix du verbe sortiri. Du reste, dans la phrase qui suit, l'intervalle de seize jours entre les idées et les calendes du mois suivant n'est pas aussi arbitraire que le laisse entendre notre auteur : il s'agit ici de la moitié du mois lunaire, mois qui préside aux divisions primitives, les calendes coïncidant avec la Nouvelle Lune, les nones avec le premier quartier et les ides avec la Pleine Lune.

${ }^{62}$ Code Justinien 3.12.3 : Omnes iudices urbanaeque plebes et artium officia cunctarum uenerabili die solis quiescant. Ruri tamen positi agrorum culturae liberi licentesque inseruiant, quoniam frequenter euenit, ut non alio aptius die frumenta sulcis aut uinae scrobibus commendentur, ne occasione momenti pereat commoditas caelesti prouisione concessa.

${ }^{63}$ Sat. I, 15, 7-8 ; trad. Ch. Guittard : «Voilà par quel hasard ils reçurent les uns trente et un, les autres vingtneuf jours. Mais, quel que fût le mois, les ides furent instituées le neuvième jour à partir des nones et l'intervalle entre les ides et les calendes suivantes fut fixé à seize jours. Aussi les mois les plus pourvus avaient-ils les deux jours qu'ils comptaient en plus entre leurs calendes et leurs nones, les uns le cinquième les autres le septième jour. Cependant César, respectant [...] l'ordre des cérémonies, ne voulut pas déplacer les nones même dans les mois auxquels il ajouta deux jours, car un scrupule religieux lui fit reléguer ses propres jours après la célébration de toutes les fêtes du mois ». 
La posture de l'auteur dans ces lignes consiste à présenter le calendrier archaïque comme un repoussoir par rapport à la révolution scientifique alliée au scrupule religieux (cf. stata sacra custodiens) qui fut l'œuvre du dictateur César. Macrobe se donne à voir, au début de ce chapitre comme un antiquaire :

ne nobis quidem, quibus origo Romana est, erubescendum puto quaerere quod quaesitu dignum omnes veteres putauerunt ${ }^{64}$.

Est-ce ce qui explique son silence sur les changements postérieurs et sur la semaine ? Ce pourrait être, pour ce païen, une façon de faire de la résistance à ces nouveaux temps qui imposent une religion nouvelle, une façon de s'opposer aux Christiana tempora.

De la même façon d'ailleurs, pour les chrétiens aussi, la semaine cristallise un certain nombre d'oppositions. Au rythme de la semaine religieuse évoquée plus haut avec Tertullien et Irénée se substitue un rythme nouveau qui ne fait plus du samedi mais du dimanche le septième jour de sanctification : «[... l'apologétique chrétienne emprunte ses arguments aux juifs expliquant la fonction du sabbat», explique Ch. Pietri, «et cela au moment où la prédiction chrétienne critique la règle sabbatique, car la pratique juive sert de repoussoir pour établir l'originalité d'une nouvelle alliance célébrant le dimanche ${ }^{65}$.

Cette intransigeance, propre à l'Italie, réussit à ne conserver, à la différence de l'Orient, aucun témoignage de célébration sabbatique.

Il en va de même de la semaine astrologique, comme le précise encore Ch. Pietri : «Pour dissiper toute équivoque les pasteurs recommandent généralement d'éviter la nomenclature astrologique des jours de la semaine. Philastre de Brescia compte cette habitude au nombre des hérésies et il accuse ceux qui imaginent originelle l'appellation de dies Saturni ou de dies Solis ».

Sans montrer autant de dureté, Augustin, par exemple, en constatant que beaucoup de chrétiens parlent du dies Mercuri, les invite à observer l'usage ecclésiastique :

\footnotetext{
${ }^{64}$ Sat. I, 15, 4 ; trad. Ch. Guittard : « [...] mais nous-mêmes, qui sommes Romains de naissance, n'avons pas à rougir, à mon sens, de recherches que l'Antiquité unanime a estimées dignes d'être entreprises ».

${ }^{65}$ Pietri, 1984, 71 .
} 
[...] feria qui Mercurii dicitur a paganis et a multis christianis [...] Melius ergo de ore christiano ritus loquendi ecclesiasticus procedit ${ }^{66}$.

On voit donc la multiplicité des luttes pour conserver l'existant ou pour faire les bons choix dans le nouveau comput du temps.

Il est temps de conclure. Au terme de cette étude, nous avons essayé de montrer que la réforme du calendrier, en général, constitue un acte éminemment politique. Pour celle de Jules César, il s'agissait, pour le dictateur, d'afficher sa domination sur l'ensemble de l'Empire romain - une domination qui se déclinait à la fois sur l'espace et sur le temps.

Quant à la division du mois, nous avons essayé de lever le voile, ou une partie du voile, sur la transition entre ancien comput des jours et semaine. Ne faisant pas l'objet d'une réforme officielle, le changement ou les réactions au changement se font de façon inapparente, voire opaque, et il convient de traquer les moindres détails significatifs. Et de quelle semaine parlet-on ? de la planétaire ou de la religieuse ? C'est là encore une autre question. Cette enquête nous a permis in fine de montrer la coexistence des deux types de comput du jour, qui, loin de s'exclure, deviennent complémentaires, car, comme le précise Ch. Pietri, « la nomenclature du cycle hebdomadaire ne sert guère à situer un événement de la vie individuelle dans le temps de la collectivité ${ }^{67}$.

${ }^{66}$ Enarr. in Ps. xciii, 3.

${ }^{67}$ Pietri, 1984, 70. 